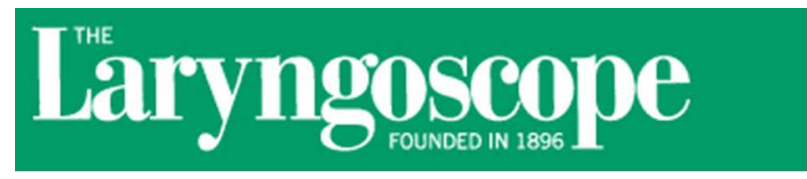

\title{
Pharmacoeconomics of Cyclamen europaeum in the management of Acute Rhinosinusitis
}

\begin{tabular}{|r|l|}
\hline Journal: & The Laryngoscope \\
\hline Manuscript ID: & Iscope-12-1797.R2 \\
\hline Wiley - Manuscript type: & Original Reports \\
\hline Complete List of Authors: & $\begin{array}{l}\text { Mullol, Joaquim; Hospital Clínic, IDIBAPS, CIBERES., Rhinology Unit \& } \\
\text { Smell Clinic, ENT Department. } \\
\text { Crespo, Carlos; Univertisty of Barcelona, Statistics; Oblikue Consulting, } \\
\text { Health Economics \& Outcomes Strategy } \\
\text { Carre, Carme; Hartington Pharmaceuticals, } \\
\text { Brosa, Max; Oblikue Consulting, }\end{array}$ \\
\hline Keywords - Combo: & $\begin{array}{l}\text { Outcomes/cost effectiveness < Clinical < Allergy/Rhinology, Nose and } \\
\text { paranasal sinuses < Head and Neck, Adult rhinology < Clinical < } \\
\text { Allergy/Rhinology }\end{array}$ \\
\hline
\end{tabular}

SCHOLARONE

Manuscripts 


\section{Pharmacoeconomics of Cyclamen europaeum in the management of Acute Rhinosinusitis}

Running title: Pharmacoeconomics of Cyclamen europaeum

Joaquim Mullol ${ }^{1}$

Carlos Crespo ${ }^{2,3}$

Carme Carré ${ }^{4}$

Max Brosa ${ }^{2}$

${ }^{1}$ Rhinology Unit \& Smell Clinic, ENT Department. Hospital Clínic, IDIBAPS, CIBERES. Barcelona, Catalonia. (Spain)

2 Oblikue Consulting, Barcelona (Spain)

${ }^{3}$ Department of Statistics. University of Barcelona, Barcelona (Spain)

${ }^{4}$ Hartington Pharmaceuticals, Barcelona (Spain)

\section{Corresponding author:}

Carlos Crespo

c/ Josep Irla i Bosch, 5-7 Primera Planta

08034-Barcelona (Spain)

Phone: +34932521377

Fax: +34932051447

Email: carlos.crespo@oblikue.com

Financial support: This study was sponsored by Hartington Pharmaceuticals.

Conflict of interest statement: Dr. J. M. contributed in this study as a clinical expert; C. C. works for Hartington Pharmaceuticals; C. C. and M. B. received a grant of Hartington Pharmaceuticals to conduct the pharmacoenomic analysis. 


\begin{abstract}
Objective. To carry out a pharmacoeconomic analysis of Cyclamen europaeum $(C E)$ in the management of acute rhinosinusitis (ARS) in Spain using data from the PROSINUS study.

Study Design. This was a prospective observational study to compare the effectiveness and cost-effectiveness between therapies including CE vs. other therapies in the management of ARS.
\end{abstract}

Methods. The study was carried out as a secondary analysis of the PROSINUS, combining healthcare resource use, productivity loses, and health outcomes from the observational study with costs representative of the Spanish Health System.

Results. CE given as monotherapy appears to be more effective (cure rate) than other monotherapies $(15.3 \%$ higher, $\mathrm{p}<0.05)$ and combination $(10.3 \%$ higher, $\mathrm{p}<0.05)$ therapies. The addition of $C E$ to other single-drug or combination therapies showed a statistically significant improvement in terms of cure rates when adding CE to 2 -drug combinations ( $93.9 \%$ vs. $76.5 \%$; $p<0.05)$, and no significant effect when added to combinations of three or more drugs ( $81.1 \%$ vs. 79.8 ; NS). CEbased therapies generally showed lower indirect costs, although only the comparison of CE alone vs. other monotherapies, with a net cost savings of $101 €$ per patient, reached statistical significance (331€ vs. 432€, $p<0.05$ ). In addition, CE-based therapies show lower cost per cured patient in all comparisons except when CE was used in combination with three or more other drugs.

Conclusions. The use of Cyclamen europaeum may be associated to better clinical outcomes at no additional cost for the healthcare system, respect to treatments commonly used for ARS in clinical practice. 
Keywords: Pharmacoeconomics, Cyclamen europaeum, acute rhinosinusitis, costs, effectiveness.

Level of Evidence: $2 c$ 


\section{INTRODUCTION}

Rhinosinusitis is an inflammatory process of the paranasal sinuses with high prevalence in clinical practice ${ }^{1,2}$. Acute rhinosinusitis (ARS) is an inflammatory alteration of the nose and paranasal sinuses. ARS is usually caused by a viral infection, although other processes such allergic rhinitis, pathological abnormalities, nasal polyps, or nasal decongestant abuse, can constitute predisposing factors. Inflammation in nasal cavity mucosa results in some disorders of the absorption, protection and transport functions, and complications like asthma flare-ups, chronification of ARS, meningitis, vision problems and ear infections. In ARS the most common symptoms experienced by patients are nasal obstruction, mucopurulent rhinorrhoea, and localized frontal or facial pain.

It is currently accepted that ARS is a frequent disease and, therefore, has a considerable impact on general public health and economic resources, both in terms of those allotted to treatment disease as well as those related to patient labor productivity. In the United States, it was estimated that in 1996 the costs that could be attributable to rhinosinusitis reached 3,390 million dollars ${ }^{3}$. The number of cases in Spain could range between 440,000 and 1,760,000 per year, with a considerable associated cost ${ }^{2}$.

ARS treatment goals should be symptomatic relief, accelerating remission and preventing complications. Indeed, there is a weak body of clinical evidence about treatment effectiveness and controlled clinical trials of ARS therapies are not numerous ${ }^{4}$. Most authors and consensus papers show little evidence on the effectiveness of treatments based on antibiotics ${ }^{5}$, oral steroids ${ }^{6}$, antihistamines, 
nasal irrigation, mucolytics and phytotherapy ${ }^{7}$. The treatment recommended for ARS by the EPOS consensus (European Position Paper on Rhinosinusitis and Nasal Polyps) ${ }^{7}$ is based on symptomatic relief (analgesics, saline serum and decongestants) or on the use of topical nasal steroids and antibiotics for the specific patients that can respond, depending on the etiology, severity and evolution of the clinical pattern ${ }^{8}$. Upon analyzing evidence of adjuvant treatment aimed to relief symptoms, it can be concluded that it is low. In Spain, the PROSINUS study ${ }^{9}$ a large observational study showed that less than one fourth (24\%) of 2,610 patients with ARS followed EPOS guidelines, and a number of different treatments and drug combinations were used.

Cyclamen europaeum has been used for a very long time, particularly in SouthEast Europe, as a traditional treatment for nasopharyngeal diseases. Cyclamen europaeum (CE) is prepared on the basis of an aqueous extract of tubers of $C E$, that contain a mixture of saponins, which characteristically causes local osmosis and stimulation of the ending terminal of the trigemin and increases the secretion of the nasal mucos ${ }^{10,11}$. CE has shown its efficacy in two placebo-controlled clinical trial and 13 head to head studies ( 9 in adults and 5 in children) that compared $C E$ with other active treatments that seem to indicate that $C E$ may contribute to reducing disease progression time, improving symptoms relief and objective response (CT-scan), decreasing the need for antibiotics or boosting their effects, as well as reducing the number of complications and chronification ${ }^{12}$.

The present study has analysed data from the PROSINUS study to carry out a 
pharmacoeconomic analysis of CE in the management of ARS in Spain.

\section{MATERIALS AND METHODS}

\section{Subjects}

The PROSINUS study included 2,610 patients visited by 287 specialists in otorhinolaryngology throughout Spain. Patients had to fulfil all inclusion criteria: either sex, $\geq 18$ years of age, reporting to the otorhinolaryngologist's office with symptoms compatible with the epidemiological diagnosis of ARS according to the EPOS consensus "sudden onset of two or more symptoms, one of which should be either nasal blockage/obstruction/congestion or nasal discharge (anterior/posterior nasal drip), \pm facial pain/pressure, \pm reduction or loss of smell; for a duration of $<12$ weeks". Patients voluntarily gave their consent to take part in this study and they had none of the exclusion criteria: established diagnosis of chronic rhinosinusitis (with or without nasal polyps, and presenting with a new event or acute exacerbation of their disease), concurrent use of medication for other conditions that could interfere with the sinonasal disease, existing social or cognitive features indicating the possibility of problems in the follow-up or a high risk of withdrawing prematurely from the study. Data on the previous management by Primary Care Physicians was also recorded. 


\section{Study design}

The PROSINUS study was an epidemiological, observational, prospective, health outcomes study aimed to describe the diagnostic, treatment and clinical monitoring procedures applied in ARS in clinical practice in Spain. Since this was an observational study aimed at identifying current practice real failure rates, and costs associated with usual medical care and not under experimental conditions, treatment of the ARS was left to the criteria of the attending physician. The present study was carried out as a secondary analysis of the PROSINUS database, combining healthcare resource use, productivity loses and health outcomes from the observational study with updated unit costs representative of the Spanish National Health System (SNHS). Data for both clinical and economic variables (see below) were recorded prospectively by the investigators (from symptoms onset until first study visit, and from first study visit to final study visit, 2 to 4 weeks after first visit).

Comparative options

Costs and outcomes of $C E$ in monotherapy and combination therapy were compared to both monotherapies and combinations therapies as follows:

- $\quad C E$ in monotherapy vs. other pooled monotherapies and vs. combinations of two or more pooled drug combinations without $C E$. 
- $\quad C E$ added to different combinations, specifically: $C E$ added to one single drug, $C E$ added to two drug combination, and $C E$ added to three or more combined drugs.

Additional analysis was carried out to address the effect of adding $C E$ to antibiotics and antibiotics + corticosteroids ${ }^{8}$.

In all cases, comparison options were defined by means of the treatments prescribed by the patients at the first visit of the study, irrespective of treatments taken previously (from event onset to first physician visit).

Clinical outcomes

Cure rate was defined following the EPOS consensus for epidemiological definition $^{7}$ as the complete clinical resolution of ARS symptoms of the ARS episode at the end of the study follow-up (visit 2 at 2-4 weeks) and was calculated for each comparison option. For the primary analysis, the patient sample that met all of the inclusion criteria and none of the exclusion criteria and which had valid data for the primary endpoint was used. No provision was made for interpolating missing data o loss to follow-up.

Economic outcomes 
Both direct and indirect costs were included in the analysis. Direct costs included medical visits (general practitioner, otorhinolaryngologist and emergency visits), diagnostic tests (rhinoscopy or nasal endoscopy, sinus X-ray, sinus CT, bacteria culture and allergy tests) and drugs (oral antibiotics, nasal corticosteroids, antihistamines, nasal decongestants, mucolytics, saline solutions, and phytotherapy including $C E$ ). Indirect costs included sick leaves (absenteeism) and reduced productivity at work (presenteeism). In the PROSINUS study, resources use from the onset of the ARS event to the date of the visit was also recorded at the first study visit. At the second visit, resources uses from first visit to the end of the event were recorded. Thus, otorhinolaryngologists could have influence in the resources use after but not before the first visit since symptoms onset.

Unit costs applied to the health resources utilization data are shown in Table 1. The data sources were the Medicine Database of the General Council of Pharmacists Official Colleges (BOTPLUS) ${ }^{13}$ for drug costs, and the Health Costs Database eSalud ${ }^{14}$ for healthcare resources (medical visits and tests). With respect to the medication, it was assumed that a patient would purchase one pack of each type of drug that he/she used. All costs were expressed in euros of 2011.

Perspective, time horizon and discount rate

The main analysis was carried out from the societal perspective (including both direct and indirect costs). Additional analysis adopted the healthcare perspective 
(direct costs only). Since study follow-up was short (1 month) no discounting was applied in the analysis.

Statistical Analysis

Statistical analysis was performed with SPSS version 15.0 for Windows (SPSS Inc., Illinois, USA). ANOVA was carried out to see if there was any difference between subgroups and logistic regression models were built to determine whether specific drugs had a significant influence in the cure rate of pooled options, to discard relevant heterogeneity of comparison options in the analysis.

Student's t-test and CHI-square test were used to compare mean costs and cure rates of CE-based therapies respect to comparators. The level of statistical significance was established at 0.05 .

\section{RESULTS}

On average, patients reported to the first PROSINUS study visit $11.2( \pm 6.2)$ days after onset of the ARS event, while the average total duration of the event was 13.7 ( \pm 8.8 ) days. At visit 2, $82.9 \%$ of patients presented complete clinical resolution of the symptomatic episode. Table 2 shows the clinical results of the comparison of CE based therapies respect to other therapies in the management of ARS. CE in monotherapy appeared to be more effective in terms of cure rate than other pooled monotherapies $(15.3 \%$ higher, $p<0.05)$ and combination therapies $(10.3 \%$ higher, $\mathrm{p}<0.05)$. The addition of $C E$ to other monotherapies or combination therapies 
showed a significant improvement when adding CE to 2-drug combinations (93.9\% vs. $76.5 \% ; p<0.05)$, but no significant effect when added to combinations of three or more drugs (81.1\% vs. $79.8 \%$; NS).

Patients receiving CE monotherapy were $98 €$ less costly than patients receiving other monotherapies (682€ vs. $584 €, p<0.05)$ and $61 €$ less costly than combination therapies (645€ vs. 584€, p<0.05). Figure 1 show the breakdown of these results in terms of direct and indirect costs. CE as an add-on treatment was associated to lower total costs when used in combination with one single drug (626€ vs. 682€, $p=0.29$ ), even though these differences didn't reach the statistical significance, probably because of the limited sample size. The combination of CE with 2 or more drugs produced similar total costs than the same combinations without CE. In all situations, cost reductions associated to CE-based therapies were mainly explained by indirect costs, whilst direct healthcare costs were similar in all subgroups (see table 3). The proportion of patients treated with CE that were visited by PCP or PCP and ORL was slightly higher than for patients not taking CE, but differences were only significant vs patients treated with antibiotics (which accounted for a small subgroup of patients). This fact could yield to higher costs for CE patients but this is not the case because of the lower number of total visits in this group.

The combination of total costs and cure rates provides an estimate of the balance between cost and outcomes, showing lower cost per cured patient in CE based therapies in almost all comparisons (except when added to combinations of 3 or more drugs) (Figure 2). 


\section{Special situations}

Since only antibiotics, either in monotherapy or combined with corticosteroids have demonstrated a clinical efficacy in the management of ARS, a specific analysis was carried out to compare these options with and without $C E$. The results of this analysis showed that adding $C E$ to one antibiotic, with or without nasal corticosteroid, increased the success rate and reduced total costs, due to a significant reduction of indirect costs. Both compared to antibiotic in monotherapy or to antibiotic plus nasal corticoid, the addition of $C E$ resulted in a lower cost per success ratio (table 4).

\section{DISCUSSION}

This secondary analysis of the PROSINUS study, an observational, prospective, health outcomes study aimed to describe the management of ARS and its outcomes, has shown that $C E$ based therapies are associated to better clinical outcomes with similar or even lower costs than comparable therapies without $C E$.

These results are in line with the conclusions of most of the clinical studies on ARS, including placebo-controlled clinical trials and head to head clinical studies. Pfaar et al., in a randomized controlled trial in patients with moderate-to-severe ARS, demonstrated that CE on the top of regular antibiotic treatment added a 
significant improvement of facial pain/pressure and signs of nasal inflammation compared to antibiotic alone ${ }^{12}$. CE is a safe and well tolerated treatment that demonstrated a great reduction in individual symptoms scores (nasal obstruction, mucus secretion, facial pain and loss of smell), an improvement in the mucosal edema and nasal obstruction evaluated by endoscopy and a reduction of the sinus occlusion evaluated by CT-can on day $7^{15}$. The effects of CE on ARS start at 3-5 days of treatment and the complete symptom resolution at 9-12 day ${ }^{15}$. CE was able to reduce the disease progression time, the need for antibiotics or boosting their effects, the number of complications and the disease chronification with a great satisfaction scores rated by patients and investigators compared to antibiotic treatment ${ }^{12}$. Several drugs, like antibiotics, oral decongestants, antihistamines, and topical corticosteroids are used for the treatment of RS but these available therapies don't have sufficient evidence to be considered efficacious and often fail to provide adequate symptoms relief and or objective cure assessed by endoscopy or CT-scan. Consequently, patients with ARS are often poorly treated and / or treated using several therapies. Despite continued development of new therapies and more accurate diagnostic procedures, there is no evidence that ARS is being better controlled. CE could be considered a recommendable option as a first line treatment as it has demonstrated in several clinical studies its ability improving the signs and symptoms of the disease. Besides, Savvateeva and Lopatin ${ }^{16}$ have recently published a review of studies that evaluated the efficacy and safety of $C E$ and concluded that it provides a good option for ARS treatment, ensuring personalized treatment and preventing polymedication and inappropriate use of antibiotics. 
As ARS frequently occurs in conjunction with asthma, allergic rhinitis, and other airway disorders, it is difficult to single out the expenditures devoted to treating rhinosinusitis only, and only a few studies have done so $^{17}$ and results are notably variable. Anzai et al. ${ }^{18}$ examined the cost-effectiveness of four treatment strategies, with and without antibiotics, for adult patients with acute sinusitis, finding a total cost per patient between $\$ 747$ and $\$ 899$ in 2005 . Bhattacharyya et al. ${ }^{19,20}$ have recently published two studies of the burden of rhinosinusitis (recurrent ARS and chronic rhinosinusitis respectively), with total cost per patient/year of $\$ 1,091$ and $\$ 780$. These results are hardly comparable with those from our study, since we focused in costs associated to a single ARS event, whilst those mentioned studies analysed longer time horizons. Finally, pharmacoeconomic analysis of two antibiotic therapies found a direct healthcare cost per ARS episode of $\$ 171-\$ 211$ in $2002^{21}$, slightly lower than our estimates of $250 €-329 €$.

Given the high prevalence of ARS and the eventual consequences of treatment failure, including potential disease chronification, clinical success should be pursued in order not only to improve patients' quality of life but to limiting the economic impact of the condition.

In our study the use of CE was associated to a higher cure rate without increasing healthcare costs, either when compared as monotherapy respect to other monotherapies, or when added to different combinations. It is important to note that not properly treated rhinosinusitis could lead to a recurrence or even to a potential chronification of the disease whereas consequently reducing patient's 
overall well-being and quality of life and increasing direct and indirect costs ${ }^{19}$. Therefore, in this sense cyclamen provides an added value because reduces chronification of ARS. Besides, cyclamen has a purely physiological effect and is not absorbed, showing a better safety profile compared to other treatments that cause more or worse adverse events and consequently produce a greater cost derived from adverse event management ${ }^{22}$.

This study has two main limitations that must be considered. First, the use of the data from an observational descriptive study to derive conclusions regarding the consequences of different treatment options is risky, i.e., the PROSINUS study was not designed to compare different therapies, and patients' characteristics may be assumed equivalent irrespective of treatment received, so a selection bias could exist. Considering the ethical and logistical weaknesses of randomised clinical trials, well designed and conducted observational studies may be an efficient alternative to explore exposure effectiveness, even though comparability across exposure groups may not be guaranteed. Second, the comparison options built in the secondary analysis include the outcomes of patients that received different drugs in different combinations, e.g., the pooled results of $C E$ vs. other monotherapies included a number of different drugs in the group 'other monotherapies'. However, logistic regression techniques were applied to address whether this pooling of clinical results were justifiable, i.e., no statistical differences between individual treatments (or combinations) were stated before aggregating patients in the pool analysis. These limitations raise the need for further research to definitely establish the clinical superiority of Cyclamen in different clinical 
situations and combined with different treatments.

Finally, it is noteworthy that the present study is pragmatic and widely applicable in clinical practice, and although it has the mentioned limitations, especially in relation to the selection of treatments, we can conclude that the trend Cyclamen europaeum has in monotherapy for ARS is cost efficient. However, further studies are needed with greater control in order to conclude on the effect of cyclamen with other treatment combinations of ARS..

\section{CONCLUSIONS}

The results of this study suggest that the use of $C E$, both as monotherapy or in combination, may be associated to better clinical outcomes, including cure rate, at no additional cost for the healthcare system, respect to different treatment options commonly used in clinical practice for the treatment of ARS. 


\section{REFERENCES}

1. Fokkens WJ. Symptoms of rhinosinusitis. Rhinology. 2011 Jun;49(2):12930.

2. Barberán MT, Ortega del Alamo $\mathrm{P}$, Mensa Pueyo $\mathrm{J}$ et al. Diagnosis and treatment of acute rhinosinusitis. Second Consensus. Rev Esp Quimioter 2008;21:45-59.

3. Kennedy DW. Overview. First line management of sinusitis: a nacional problem? Otolaryngol Head Neck Surg. Suppl 1990; 103: 847-854.

4. Wang $D-Y, R$ Wardani, $K$ Singh et al. A survey on the management of acute rhinosinusitis among Asian physicians. Rhinology 2011; 49: 264-71.

5. Burton MJ, Kuppersmith RB, Rosenfeld RM. Extracts from The Cochrane Library: Antibiotics for acute maxillary sinusitis. Otolaryngol Head Neck Surg 2008;139:486-9.

6. Lous J. Nasal steroids for acute sinusitis. A survey of a Cochrane review. Ugeskr Laeger 2008;170:2869-71.

7. Fokkens WJ, Lund VJ, Mullol J. European Position Paper on Rhinosinusitis and nasal Polips Group. European Position Paper on Rhinosinusitis and Nasal Polyps 2007. Rhinology 2007;45(Suppl. 20):1-139.

8. Meltzer EO, Bachert C. Staudinger H. Treating acute rhinosinusitis: comparing efficacy and safety of mometasone furoate nasal spray, amoxicillin, and placebo. J Allergy Clin Immunol 2005;116: 1289-95. 
9. Montserrat-Gili JR, J Mullol. Prospective epidemiological study of the diagnosis and therapeutic management of acute rhinosinusitis in Spain. ERS\&ISIAN Abstract Book 2008: 60-61. [Abstract]

10. Gedevanishvili MD, Gogitidze NM, Sikharulidze IS. Reflex mechanisms of nasoparanasal secretion in administration of sinuforte. Vestn Otorinolaringol 2007;3:54-6.

11. Lopatin AS, Shempelev OA. Influence of Sinuforte on the blood microcirculation in the nasal cavity mucosa. Russian Rhinology 2007; 4:2628.

12. Pfaar $\mathrm{O}$, Mullol J, Anders $\mathrm{C}$ et al. Cyclamen europaeum nasal spray, a novel phytotherapeutic product for the management of acute rhinosinusitis: a randomized double-blind, placebo-controlled trial. Rhinology. 2012 Mar;50(1):37-44

13. Medicine data base of the General Council of Pharmacists Official Colleges. BOTPLUS. Available at: http://www.portalfarma.com [Last accessed 21 December 2010].

14. eSalud. Spanish Health Costs database. Oblikue Consulting. 2010. Available at: http://www.oblikue. com/bddcostes/ [Last accessed 21 December 2010].

15.Ponikau JU, Hamilos DJ, Barreto A, Cecil J, Jones SW, Manthei SE. Efficacy and Safety of a Cyclamen europaeum extract nasal spray in acute sinusitis: a randomized clinical trial. Poster 2170. 1st CEORL-HNS. Barcelona 2011. 
16. Savvateeva DM, Lopatin AS. Application of sinuforte in domestic and foreign practices. Vestn Otorinolaringol 2010;1:88-92.

17.Wasserfallen JB, Livio F, Zanetti G. Acute Rhinosinusitis A Pharmacoeconomic Review of Antibacterial Use. Pharmacoeconomics 2004; 22: 829-837.

18. Anzai Y, Jarvik JG, Sullivan SD, Hollingworth W. The cost-effectiveness of the management of acute sinusitis. Am J Rhinol. 2007 Jul-Aug;21(4):44451.

19. Bhattacharyya N, Grebner J, Martinson NG. Recurrent Acute Rhinosinusitis: Epidemiology and Health Care Cost Burden. Otolaryngol Head Neck Surg. 2011 Oct 25 doi: 10.1177/0194599811426089.

20. Bhattacharyya N, Orlandi RR, Grebner J, Martinson M. Cost burden of chronic rhinosinusitis: a claims-based study. Otolaryngol Head Neck Surg. $2011 ; 144: 440-5$

21. Keating KN, Friedman HS, Perfetto EM. Moxifloxacin versus levofloxacin for treatment of acute rhinosinusitis: a retrospective database analysis of treatment duration, outcomes, and charges. Curr Med Res Opinion 2008; $22: 327-333$.

22. Demoly $P$. Safety of intranasal corticosteroids in acute rhinosinusitis. Am J Otolaryngol 2008;29:403-13. 
Figure 1. Results of the cost analysis - CE monotherapy vs other monotherapies (pooled) and combinations

Figure 2. Total cost per cured patient

Legend: CE: Cyclamen europaeum 
Table 1. Unit costs used in the analysis

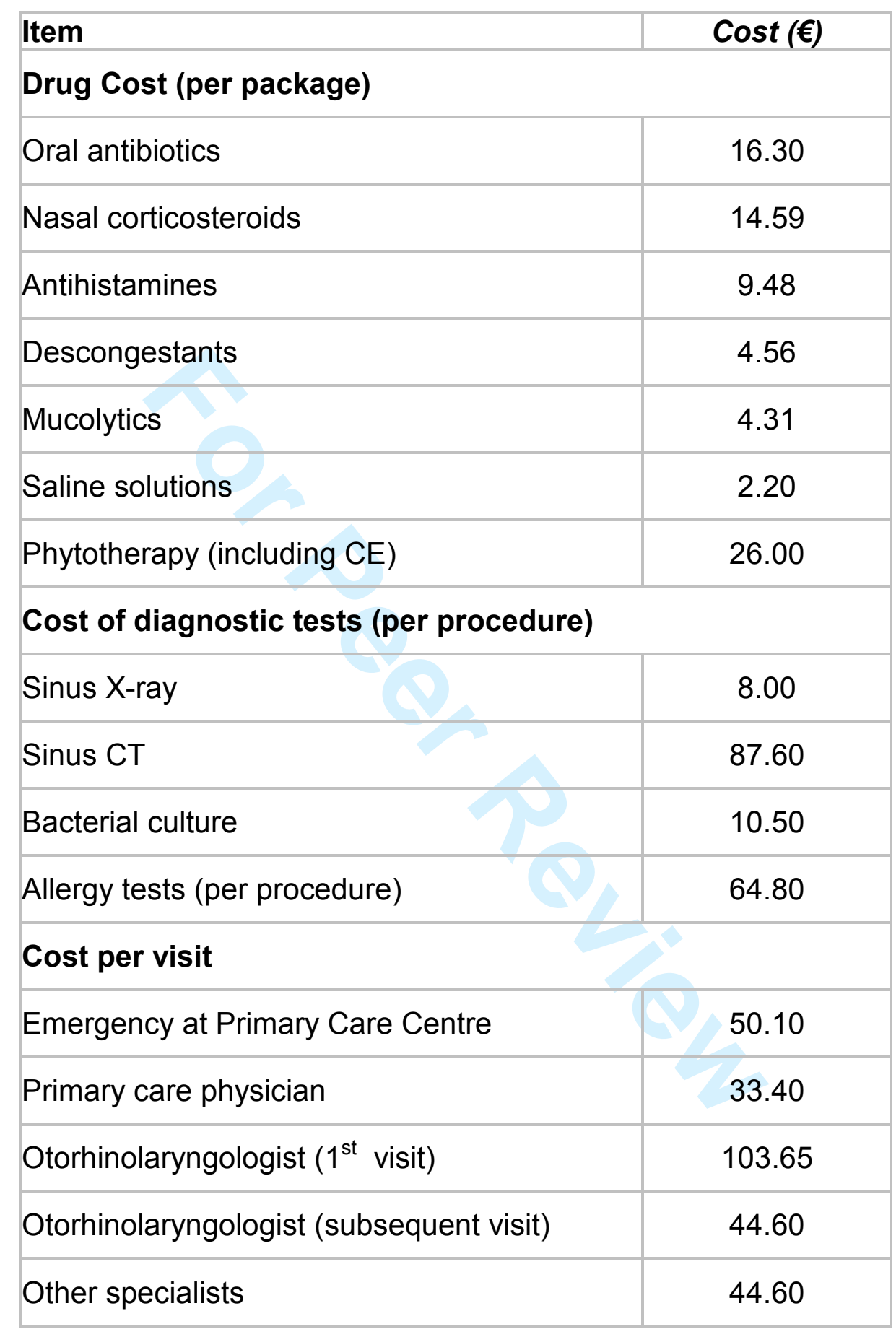

Sources: BOTPLUS 2011, eSalud 2011. CE: Cyclamen europaeum; CT: computerized tomography 
Table 2. Cure rates of Cyclamen-based options vs. other treatment options (comparators).

\begin{tabular}{|c|c|c|c|c|}
\hline Comparison & $\begin{array}{c}\text { Cyclamen } \\
\% \text { mean (SD) }\end{array}$ & $\begin{array}{c}\text { Comparator } \\
\% \text { mean (SD) }\end{array}$ & $\begin{array}{c}\text { Difference } \\
\%\end{array}$ & p-value \\
\hline $\begin{array}{l}\text { Cyclamen } \\
\text { monotherapy } \\
(n=90) \text { vs. } \\
\text { Other } \\
\text { monotherapies } \\
\text { (pooled) }(n=68)\end{array}$ & $88.8(31.8)$ & $73.5(44.4)$ & +15.3 & $<0.05$ \\
\hline $\begin{array}{l}\text { Cyclamen } \\
\text { monotherapy } \\
(\mathrm{n}=90) \mathrm{vs} . \\
\text { Other } \\
\text { combinations } \\
\text { (pooled) } \\
(\mathrm{n}=221)\end{array}$ & $88.8(31.8)$ & $78.5(41.1)$ & +10.3 & $<0.05$ \\
\hline $\begin{array}{l}\text { Cyclamen } \\
(\mathrm{n}=61)+1 \text { drug } \\
\text { (pooled) vs. } 1 \\
\text { single drug } \\
\text { (pooled) }(\mathrm{n}=68)\end{array}$ & $81.7(39.0)$ & 73.5 (44.4) & +8.2 & NS \\
\hline $\begin{array}{l}\text { Cyclamen } \\
(\mathrm{n}=82)+2 \\
\text { drugs } \\
\text { combinations } \\
\text { (pooled) vs. } 2 \\
\text { drugs } \\
\text { combinations } \\
\text { (pooled) }(\mathrm{n}=87)\end{array}$ & $93.9(24.1)$ & $76.5(42.7)$ & +17.4 & $<0.05$ \\
\hline $\begin{array}{l}\text { Cyclamen }+3 \\
\text { or more drug } \\
\text { combinations } \\
\text { (pooled) }(n=86) \\
\text { vs. } 3 \text { or more } \\
\text { drug } \\
\text { combinations } \\
\text { (pooled) } \\
(n=134)\end{array}$ & $81.1(40.0)$ & $79.8(39.0)$ & +1.3 & NS \\
\hline
\end{tabular}

NS: non-significant; SD: standard deviation 
Table 3. Results of the cost analysis. CE in combination

\begin{tabular}{|c|c|c|c|c|}
\hline & $\begin{array}{l}1 \text { single drug } \\
\quad(n=68)\end{array}$ & $\begin{array}{l}\text { Cyclamen }+1 \\
\text { drug }(n=61)\end{array}$ & Difference & $p$-value \\
\hline \multirow{4}{*}{\begin{tabular}{|r|} 
Total costs, mean \\
(SD) \\
Direct \\
Indirect
\end{tabular}} & $682 €(233 €)$ & $626 €(359 €)$ & $-56 €$ & NS \\
\hline & $250 €(135 €)$ & $269 €(108 €)$ & $+19 €$ & NS \\
\hline & $432 €(193 €)$ & $357 €(328 €)$ & $-75 €$ & NS \\
\hline & $\begin{array}{l}2 \text { drugs } \\
(n=87)\end{array}$ & $\begin{array}{c}\text { Cyclamen } \\
+2 \text { drugs }(n=82)\end{array}$ & Difference & $p$-value \\
\hline \multirow[t]{4}{*}{$\begin{array}{l}\text { Total costs, mean } \\
\text { (SD) }\end{array}$} & $628 €(235 €)$ & $629 €(245 €)$ & $+1 €$ & NS \\
\hline & $258 €(S D 107 €)$ & $290 €($ SD $89 €)$ & $+32 €$ & NS \\
\hline & $370 €(S D 205 €)$ & $339 €(S D 209 €)$ & $-31 €$ & NS \\
\hline & $\begin{array}{l}3 \text { or more drugs } \\
\quad(n=134)\end{array}$ & $\begin{array}{c}\text { Cyclamen }+3 \text { or } \\
\text { more drugs } \\
(n=86)\end{array}$ & Difference & p-value \\
\hline $\begin{array}{l}\text { Total costs, mean } \\
\text { (SD) }\end{array}$ & $656 €(1089 €)$ & $691 €(263 €)$ & $+34 €$ & NS \\
\hline Direct Costs & $278 €(182 €)$ & $329 €(464 €)$ & $+50 €$ & NS \\
\hline Indirect Costs & $378 €(616 €)$ & $362 €(367 €)$ & $-16 €$ & NS \\
\hline
\end{tabular}

NS: non-significant; SD: standard deviation 
Table 4. Costs and outcomes of adding CE to antibiotic with or without corticosteroids

\begin{tabular}{|r|c|c|c|c|}
\hline & $\begin{array}{c}\text { Antibiotic } \\
(\mathrm{n}=14)\end{array}$ & $\begin{array}{c}\text { CE }+ \text { Antibiotic } \\
(\mathrm{n}=24)\end{array}$ & Difference $p$-value \\
\hline Total costs, mean (SD) & $686 €(233 €)$ & $479 €(261 €)$ & $-206 €$ & $<0.05$ \\
\hline Direct & $247 €(97 €)$ & $255 €(117 €)$ & $+8 €$ & NS \\
\hline Indirect & $439 €(201 €)$ & $224 €(223 €)$ & $-214 €$ & $<0.05$ \\
\hline Cure rate, mean (SD) & $60.0 \%(50.7)$ & $91.3 \%(28.8)$ & $+31.3 \%$ & NS \\
\hline Cost per cured patient & $1.143 €$ & $525 €$ & & \\
\hline & & & & \\
\hline Dotal costs, mean (SD) & $648 €(194 €)$ & $650 €(182 €)$ & $+2 €$ & NS \\
\hline Direct & $292 €(95 €)$ & $292 €(57 €)$ & $0 €$ & NS \\
\hline Indirect & $355 €(356 €)$ & $358 €(187 €)$ & $-2 €$ & NS \\
\hline Cure rate, mean (SD) & $57.0 \%(51.0)$ & $100 \%(0)$ & & \\
\hline Cost per cured patient & $1.134 €(816 €)$ & $650 €(182 €)$ & & \\
\hline & Corticosteroid & Corticosteroid & & \\
\hline & $(n=14)$ & $(n=19)$ & & \\
\hline & & & & \\
\hline & & & & \\
\hline & & & & \\
\hline & & & & \\
\hline
\end{tabular}

NS: non-significant; SD: standard deviation. 


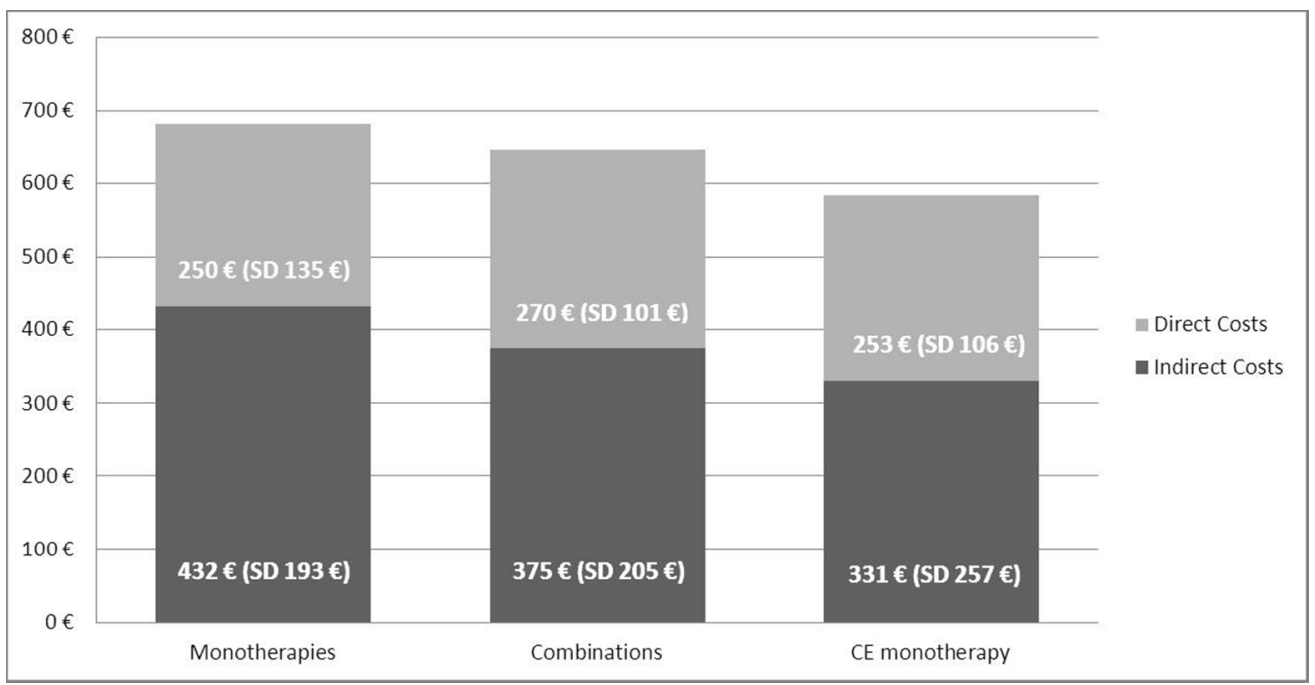

Results of the cost analysis - CE monotherapy vs other monotherapies (pooled) and combinations. $195 \times 101 \mathrm{~mm}(150 \times 150 \mathrm{DPI})$ 
